\title{
Phonostyle et réduction vocalique en français laurentien
}

\author{
Mélanie Lancien ${ }^{1, *}$, et Marie-Hélène Côté ${ }^{1}$ \\ ${ }^{1}$ Section SLI, Faculté des Lettres de l'Université de Lausanne, 1015 Lausanne, Suisse
}

Résumé. Cet article étudie le lien entre réduction vocalique et phonostyle en français laurentien en suivant une méthodologie proche de celle de Harmegnies et Poch-Olivé [1992,1994]. Nous avons analysé 9748 voyelles, produites par 10 locutrices issues du corpus PFC-Canada. Ces voyelles ont été produites dans trois styles de parole différents : la lecture, une conversation avec un enquêteur et une conversation avec un membre de la famille. Nous avons comparé la durée, la centralisation, la dispersion intra-catégorie et la diffusion des voyelles en fonction du style de parole. Nos résultats montrent un impact de la tâche de production (lecture/discussion), ainsi qu'un impact de la relation entre le locuteur et l'interlocuteur (inconnu/famille) sur la réalisation acoustique des voyelles. Les voyelles sont plus courtes et plus dispersées au sein de leur catégorie, et le système plus centralisé, en parole spontanée qu'en lecture. Elles sont également plus courtes et plus dispersées au sein de leur catégorie lors d'une discussion avec un membre de la famille que lors d'une discussion avec un enquêteur inconnu.

\begin{abstract}
Phonostyle and vowel reduction in Laurentian French. This paper studies the link between vowel reduction and phonostyle in Laurentian French, following a methodology similar to that of Harmegnies and Poch-Olivé [1992, 1994]. We analyzed 9748 vowels, produced by 10 female speakers from the PFC-Canada corpus. These vowels were produced in three different speech styles: reading, a conversation with an investigator, and a conversation with a family member. We compared the duration, the centralization, the intra-category dispersion, and the diffusion of these vowels according to the style of speech. Our results show an impact of the production task (reading/discussion), as well as an impact of the relationship between the speaker and the interlocutor (unknown/family) on the acoustic realization of vowels. Vowels are shorter and more dispersed within their category, and the system is more centralized, in spontaneous speech than in reading. They are also shorter and more dispersed within their category in a discussion with a family member than in a discussion with an unknown investigator.
\end{abstract}

\footnotetext{
*Corresponding author : melanie.lancien@unil.ch
} 


\section{Introduction}

\subsection{Les facteurs de variation dans la parole}

La variation dans le flux sonore est un phénomène connu depuis les premières expérimentations de l'Abbé Rousselot [1897], qui, grâce au kymographe, a pu démontrer qu'acoustiquement les sons prononcés n'étaient jamais exactement les mêmes. En parallèle, les études de Passy [1917] ont posé la distinction entre un style de parole « soignée » et un style de parole « familière » qui diffèrent du point de vue lexical, syntaxique et phonétique. L'intérêt pour ces variations se constituera par la suite en un sous-domaine de la linguistique appelé phonostylistique [Léon, 1993].

Telle qu'elle a été décrite par Léon [1993], la phonostylistique est « l'étude de la variabilité phonique, essentiellement humaine, et celle de sa perception en tant qu'information supplémentaire » [Léon, 1993, p.6], soit l'étude de la variation créée par des éléments extra-linguistiques tels que les différents paramètres d'une situation de communication, dont font partie l'interlocuteur et ses besoins. La sociolinguistique, représentée par Labov $[1972 ; 2002]$, apporte une pierre fondamentale à la poursuite de ces investigations, dans le sens où ses travaux posent une instrumentalisation de la variation : l'alternance linguistique, comme il l'appelle, est le fait qu'un locuteur varie son utilisation de la langue en fonction de trois critères :

- Les relations entre le destinateur et le destinataire (relation filiale, amicale, professionnelle, avec ou sans notion hiérarchique, ...)

- Le contexte social (entretien formel, discussion privée, conférence, ...)

- Le thème du discours (opinion politique, anecdote de vacances, ...). À ces critères Eskenazi [1993] ajoutera :

- Le taux d'intelligibilité (endroit bruité, problèmes d'audition, ...)

- La couche sociale (différence de classe sociale entre les interlocuteurs)

L'une des premières recherches à avoir étudié la variation stylistique, ou phonostylistique, du point de vue de la phonétique expérimentale est celle de Bernard Harmegnies et Dolors Poch-Olivé, qui ont montré des différences dans l'espace acoustique occupé par les voyelles, qui est plus réduit en parole spontanée qu'en parole de laboratoire [1992]. Harmegnies et Poch-Olivé [1994] ont par la suite comparé, dans une étude de cas, six situations de communication différentes, qu'ils regrouperont en trois grandes familles de styles homogènes sur la base de la dispersion/réduction de l'espace vocalique du locuteur. Ces trois familles, pouvant être identifiées comme trois phonostyles, sont 1) la lecture, 2) la conversation spontanée, 3) la description/explication d'image. Chacun de ces styles implique des besoins différents pour la réussite de la tâche de communication et il apparaît que l'extension/réduction de l'espace vocalique s'adapte à ces besoins.

Rouas et collègues [2010], à la suite de Nakamura et collègues [2008], ont également constaté, dans une comparaison quantitative de la parole préparée (journalistique), la conversation spontanée et la parole lue, une plus forte réduction spectrale et temporelle en parole préparée qu'en parole lue et une plus grande réduction en parole conversationnelle qu'en parole préparée.

De nombreux facteurs peuvent influencer la réalisation d'une voyelle en parole continue, a fortiori dans une situation de communication spontanée. Ces modifications sont souvent produites à des fins communicationnelles, comme le démontre le modèle «HypoHyper » de Lindblom [1990]. Les locuteurs d'une langue vont, selon ce modèle, varier la clarté de leur élocution en fonction des informations nécessaires à l'interlocuteur pour le bon déroulement de l'interaction. Ainsi lorsque l'interlocuteur a besoin d'un maximum d'informations acoustiques pour comprendre le message le locuteur va hyperarticuler; a contrario, si l'interlocuteur peut combler le manque d'informations acoustiques grâce à 
d'autres informations (en général liées au contexte de communication), le locuteur va réduire son effort articulatoire et hypoarticuler.

La variation décrite ci-dessus est donc due à des facteurs extra ou paralinguistiques. Lindblom propose comme hypothèse qu'elle soit intimement liée à l'interlocuteur et au contexte dans lequel se déroule l'interaction. Ce phénomène d'adaptation à l'interlocuteur avait, avant Lindblom, été noté par Bell ou Flikeid et Cichocki, qui observaient qu'un annonceur radio variait son emploi des variétés standards et non standards de la langue en fonction du public ciblé [Bell, 1984] ou que les Acadiens utilisaient moins la variété acadienne du français en présence d'un interlocuteur originaire d'une autre communauté linguistique [Flikeid et Cichocki, 1987]. Cependant, ce n'est qu'au début des années 2000 que les premières études sur la forme sonore, sur l'acoustique de la langue, se penchent sur la question de l'adaptation à l'interlocuteur. C'est ainsi que Bradlow et collègues [2003] mettent en évidence, pour l'anglais, le fait que les locuteurs modifient les caractéristiques spectrales des phonèmes qu'ils prononcent lorsqu'ils s'adressent à des enfants ayant des difficultés linguistiques: les différences articulatoires entre les phonèmes sont volontairement exagérées pour aider l'enfant à comprendre (hyperarticulation). Ce phénomène, que la littérature retiendra sous le nom de «clear speech », sera étudié plus en détail par la suite, notamment par Rebecca Scarborough [Scarborough et. al, 2007 ; 2013 ; Scarborough, 2013]. Elle montrera notamment des différences importantes de l'espace acoustique sur un plan F1-F2, en fonction de la relation interpersonnelle (Friend), du fait qu'un mot soit énoncé en situation de communication réelle ou dans sa forme de citation (Real vs Citation) et d'éventuels problèmes auditifs de l'interlocuteur $(\mathrm{HoH}-$ hard of hearing -). La figure 1 schématise le placement de chaque situation sur le continuum allant de l'hypoarticulation à l'hyperarticulation. Ces résultats ont été obtenus par l'analyse de la position des deux premiers formants de 1266 exemplaires de voyelles présentes dans des mots cibles expérimentalement contrôlés, ainsi que de la durée de ces voyelles.
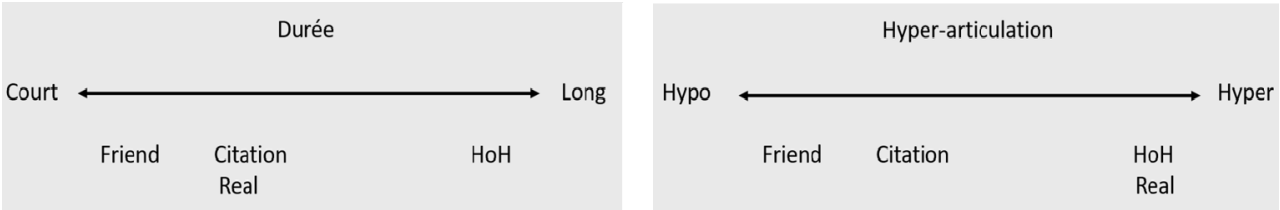

Fig. 1. : Schémas récapitulatifs des continuums d'hyper-hypo articulation mis en évidence par Scarborough et Zellou [2013] dans 4 situations de communication « Friend »= parler comme à un ami ; « Real »= tâche de remplissage de document interactif en duo (entre deux participants volontaires) ; «Citation » = forme de citation de mot ; « $\mathrm{HoH} »($ hard of hearing) = parler comme si vous deviez être compris par quelqu'un ayant des problèmes d'audition.

\subsection{L'inventaire vocalique du français laurentien}

Le système vocalique du français laurentien, repris de Côté [2012], compte 15 voyelles orales, représentées sur la trapèze vocalique schématisé en figure 2 . À ces 15

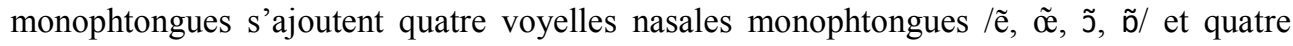
diphtongues ouvrantes /ui, wb, wa, wẽ/. 


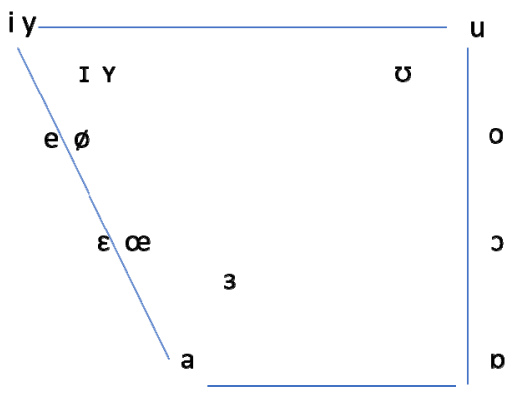

Fig. 2. : Schématisation du trapèze des voyelles orales du français laurentien selon Côté [2012].

Dans cette variété, comme en français standard, l'accent lexical tombe sur la dernière syllabe. On distingue deux groupes de voyelles selon leur propension à l'allongement et à la diphtongaison. Les voyelles $[\mathrm{i}, \mathrm{y}, \mathrm{u}, \mathrm{e}, \varnothing, \mathrm{o}, 3, \mathrm{p}, \mathrm{wp}]$ et les voyelles nasales s'allongent en syllabe finale fermée et en position prétonique (les voyelles fermées prétoniques faisant

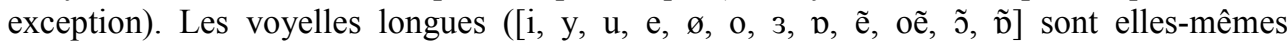
soumises à la diphtongaison, qui consiste à ajouter à la voyelle la glissante de même antériorité et arrondissement. La diphtongaison est régulière en syllabe finale fermée et plus rare en position prétonique; elle affecte également certaines voyelles en syllabe finale ouverte. La diphtongaison représente selon [Walker, 1984] un renforcement supplémentaire d'un segment déjà renforcé par la longueur. Les voyelles toniques (finales de mot) et prétoniques sont donc en position forte sur un plan phonologique, ce qui les rend théoriquement moins sujettes à la centralisation (cf. [Johnson, 2004 ; Tiffany, 1959 ; Van Bergem, 1993]).

\section{Objectifs et Hypothèses}

Cette étude vise à décrire les phénomènes de réduction vocalique selon le style de parole en français laurentien, en opposant, d'une part, la lecture et la parole spontanée et, d'autre part, la conversation avec un enquêteur inconnu et celle avec un interlocuteur familier.

Nous posons l'hypothèse que, tout comme l'anglais et le français parisien déjà mentionnés, le français laurentien montre une centralisation du système vocalique en parole spontanée, un contexte où l'hypoarticulation est attendue, par opposition à la lecture. En plus de cette centralisation globale du système, nous postulons que la réduction vocalique sera également visible dans la dispersion intra-catégorie [Audibert et. al, 2015], dans la durée des segments et dans la distance entre les deux premiers formants [Erickson, 2002] (les voyelles compactes seraient ainsi plus diffuses et les diffuses plus compactes en parole spontanée).

Au-delà d'une simple différence de degré d'hypo-articulation entre la lecture et la parole spontanée, nous postulons également une plus grande hypoarticulation et donc une plus grande réduction vocalique lorsque l'interlocuteur est un membre de la famille que lorsque c'est un inconnu (en l'occurrence un enquêteur). Ainsi la proximité relationnelle et la formalité de la discussion influenceraient le degré d'hypoarticulation.

\section{Méthode}

La présente étude s'appuie sur les données du corpus PFC-Canada [Côté, 2014], récolté dans le cadre du projet Phonologie du Français Contemporain [Durand et. al, 2002]. 


\subsection{PFC-Canada : protocole d'enregistrement}

Le protocole mis au point pour l'ensemble du projet PFC comprend deux tâches de lecture à voix haute (liste de mots et texte) et deux tâches de parole spontanée : un entretien semidirigé ( «conversation guidée ») et un entretien non dirigé ( « conversation libre »). Le corpus PFC est composé de différents points d'enquête, chacun comprenant une douzaine de locuteurs également répartis entre hommes et femmes et trois groupes d'âge.

Nous considérons ici le sous-corpus québécois de PFC, qui contient 30 points d'enquête au Québec, auxquels s'ajoutent deux points en Ontario et au Nouveau-Brunswick situés à la frontière avec le Québec, pour un total de 447 locuteurs. Toutes ces enquêtes ont été réalisées sous la responsabilité de la seconde auteure, à l'exception de la première enquête, complétée par Luc Baronian à Saguenay. Les participants sont tous originaires de la communauté visée et y ont passé la plus grande partie de leur vie. Afin de maximiser la différence entre les conversations guidée et libre, la première implique normalement le participant seul avec un enquêteur inconnu, la seconde entre deux et quatre participants de la communauté (entre un et trois participants à l'enquête, plus éventuellement un enquêteur). À la liste de 94 mots incluse dans le protocole PFC général, le sous-corpus canadien ajoute une seconde liste de 209 ou 210 items visant à documenter les traits de prononciation spécifiques des variétés canadiennes. Cette liste comprend 209 ou 210 items dans les enquêtes réalisées par la seconde auteure, dont seront tirées toutes les données retenues pour cette étude (voir section 3.3).

Les enregistrements ont généralement été réalisés au domicile du locuteur interviewé, dans une pièce aussi calme que possible, grâce à différents types d'enregistreurs numériques à microphone intégré.

\subsection{Traitement des données}

Le projet PFC a mis en place une transcription orthographique des données recueillies alignée au signal sonore sous Praat [Boersma, 2006]. Cette transcription prévoit principalement le découpage du continuum sonore en tours de parole en fonction des pauses silencieuses et du changement de locuteur, avec une transcription des mots selon leur forme orthographique standard. Les chevauchements de parole sont indiqués entre chevrons et la parole est transcrite sans correction (un « ne » de négation ne sera donc transcrit que s'il est réalisé).

À la transcription orthographique, nous avons ajouté une segmentation en phones, obtenue de façon automatique par une version adaptée pour le français du Penn Forced Aligner [Milne, 2014]. Les frontières des phones ciblés dans cette étude ont été révisées manuellement. Les phones vocaliques ont été segmentés au début et à la fin du second formant. Chaque voyelle a été identifiée par sa catégorie phonémique, de même que la consonne précédente et la consonne suivante. Les valeurs formantiques ont été extraites automatiquement grâce à un script Praat qui calcule la moyenne des fréquences de F1 et F2 à partir de trois valeurs extraites à chaque tiers du segment. Ces valeurs ont ensuite été filtrées de façon à identifier les erreurs de détection, qui ont été corrigées à la main par un examen de la coupe spectrale du segment. La durée et le contexte consonantique du phone ont été extraits par le même script.

\subsection{Sous-corpus pertinent}

Le sous-corpus choisi pour cette étude comporte 10 locutrices natives du français laurentien ( + , âge moyen $\approx 40 \mathrm{ans}$ ), enregistrées entre 2010 et 2013 dans les villes de Grande-Rivière (code d'enquête cqg), Saint-Éphrem-de-Beauce (cqj), Montréal (cqc), Hawkesbury (coc) et La Pocatière (cqf). Ces locutrices ont toutes fait la conversation guidée avec un·e 
enquêteur'trice inconnu $\cdot$ e et la conversation libre avec une seule autre locutrice membre de leur famille (sœur ou nièce). Les conversations libres durent environ 46 minutes par locutrice et les guidées 20 minutes, pour un total de 8,7 heures de parole spontanée en interaction (six des locutrices étant impliquées dans trois conversations libres). Les listes de mots représentent environ 92 minutes de données.

Dans le but de contrôler le plus possible le matériel recueilli nous avons extrait toutes les voyelles situées en syllabe ouverte de mot lexicaux bisyllabiques. Les deux dernières syllabes se trouvent en position forte en français laurentien, la pénultième a fortiori si elle est également initiale, comme c'est le cas ici. Les voyelles sont ainsi plus longues et moins sujettes à la réduction undershoot. Cette sélection nous a permis d'extraire de ce corpus

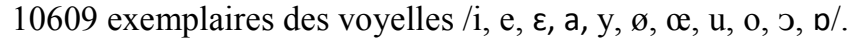

Les phonèmes $/ \varnothing$, $\propto /$ et $/ y /$ étant hautement sous-représentés, nous les avons évincés de façon à maintenir un certain équilibre dans le design expérimental, nous laissant ainsi avec un total de 9748 exemplaires vocaliques. De même, pour des questions de représentativité, nous avons regroupé les voyelles mi-ouvertes et mi-fermées antérieures $(/ \varepsilon, \mathrm{e} /)$ en une seule catégorie, ainsi que les mi-ouvertes et mi-fermées postérieures $(/ \mathrm{O}, \mathrm{o} /)$ et les deux voyelles ouvertes $(/ \mathrm{a}, \mathrm{b} /)$. Les tableaux 1 et 2 montrent le nombre de voyelles retenues par locutrice et par style de parole.

Tableau 1. Nombre de phone de chaque catégorie produit par chaque locutrice.

\begin{tabular}{cccccccccccc} 
Locutrice & COC & CQC & CQF & CQG & CQG & CQG & CQG & CQG & CQG & CQJ & Grand \\
/Phonème & JG & MC & FG & DB & JC & KD1 & KD2 & MC & SB & LM & Total \\
\hline /A/ & 209 & 265 & 156 & 328 & 195 & 227 & 212 & 256 & 83 & 249 & 2180 \\
/E/ & 426 & 485 & 313 & 522 & 388 & 392 & 370 & 493 & 175 & 464 & 4028 \\
/i/ & 122 & 59 & 109 & 164 & 113 & 139 & 160 & 149 & 54 & 141 & 1210 \\
/O/ & 195 & 120 & 139 & 179 & 154 & 152 & 128 & 203 & 67 & 129 & 1466 \\
/u/ & 116 & 85 & 61 & 98 & 92 & 83 & 74 & 120 & 42 & 93 & 864 \\
\hline Grand & & & & & & & & & & & \\
Total & 1068 & 1014 & 778 & 1291 & 942 & 993 & 944 & 1221 & 421 & 1076 & $\mathbf{9 7 4 8}$
\end{tabular}

Tableau 2. Nombre d'exemplaire de chaque catégorie produit par style de parole.

\begin{tabular}{ccccc|}
$\begin{array}{c}\text { Style/ } \\
\text { Phonème }\end{array}$ & GUIDEE & LIBRE & LISTE & Grand Total \\
\hline /A/ & 807 & 1204 & 169 & 2180 \\
/E/ & 1588 & 2068 & 372 & 4028 \\
/i/ & 397 & 603 & 210 & 1210 \\
/O/ & 474 & 783 & 209 & 1466 \\
/u/ & 333 & 438 & 93 & 864 \\
\hline Grand Total & 3599 & 5096 & 1053 & $\mathbf{9 7 4 8}$ \\
\hline
\end{tabular}

Le contexte consonantique ayant un fort impact sur la variation formantique, nous avons également cherché à le contrôler, ou du moins calculer le poids de cette variable. Les voyelles recueillies sont situées dans 65 contextes consonantiques différents, les contextes 
les plus représentés étant les suivants : coronale_coronale (1836 exemplaires), coronale_bilabiale (1239 exemplaires), bilabiale_coronale (945 exemplaires), coronale \# (727 exemplaires), coronale_labio-dentale (634 exemplaires) et uvulaire_bilabiale (404 exemplaires).

\subsection{Métriques étudiées}

L'objectif de cette étude étant d'observer les phénomènes de distorsion et de réduction de l'espace vocalique du français laurentien, nous avons extrait les premier et deuxième formants $(\mathrm{Hz})$ de chaque exemplaire vocalique retenu pour les analyses. Les valeurs en Hertz ont ensuite été normalisées en Bark, comme conseillé par [Nguyen et Adda-Decker, 2013] pour l'étude phonétique des grands corpus oraux ; cette échelle correspondant mieux à ce qui est perçu par l'auditeur. Ces deux formants ont ensuite permis, grâce à des calculs de distance euclidienne, le calcul de la distance de chaque exemplaire par rapport au centre virtuel du système vocalique laurentien (équation 3), sa distance par rapport au centre de sa catégorie vocalique (équation 2) et la diffusion de la voyelle (équation 1) [Erickson, 2002]. Nous avons également extrait la durée de chaque exemplaire (ms).

$$
\begin{gathered}
C=F 2-F 1 \\
d=\sqrt{ }(\text { F1token }- \text { F1centre_type })^{2}+(\text { F2token }- \text { F2centre_type })^{2} \\
d=\sqrt{ }(\text { F1token }- \text { F1centre_système })^{2}+(\text { F2token }- \text { F2centre_système })^{2}
\end{gathered}
$$

L'équation (3) ainsi que la figure 3 illustrent et expliquent le concept de distance d'un exemplaire par rapport au centre du système vocalique auquel il appartient. Dès les premières études de Harmegnies et Poch-Olivé [1992] jusqu'à celle de Fougeron et Audibert [2011], dont nous avons repris les méthodes de calcul, cette mesure a été utilisée pour quantifier et observer le processus de réduction spectrale des voyelles. Lindblom [1963] précisait tout de même que cette mesure ne devait pas être considérée comme la preuve d'une modification de la dynamique articulatoire.

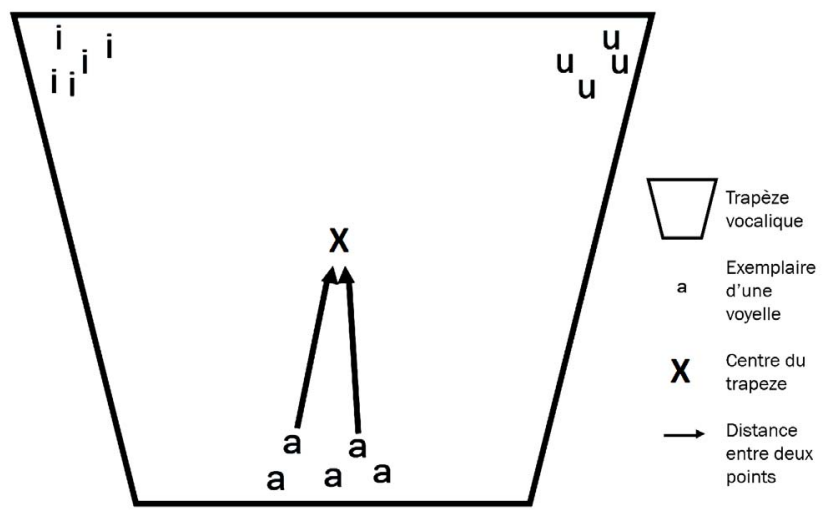

Fig. 3. Schématisation simplifiée de la méthode de calcul de la distance d'un exemplaire par rapport au centre du système vocalique.

La plupart des études sur la réduction vocalique se sont contentées de cette mesure dite de « centralisation ${ }^{i}$; des recherches plus récentes ont cependant montré que d'autres mesures étaient parfois plus pertinentes pour décrire la perte d'information spectrale [Ferguson et 
Kewley-Port, 2007 ; Fougeron et Audibert, 2011 ; Audibert et. al, 2015]. La dispersion intra-catégorie est l'une de ces mesures : calculée sur le même principe que la centralisation du système, elle permet la mesure de la distance euclidienne entre chaque exemplaire d'une catégorie vocalique et le centre de cette catégorie vocalique. L'équation (2) et la figure 4 illustrent le concept de distance d'un exemplaire par rapport au centre de la catégorie phonologique à laquelle il appartient.

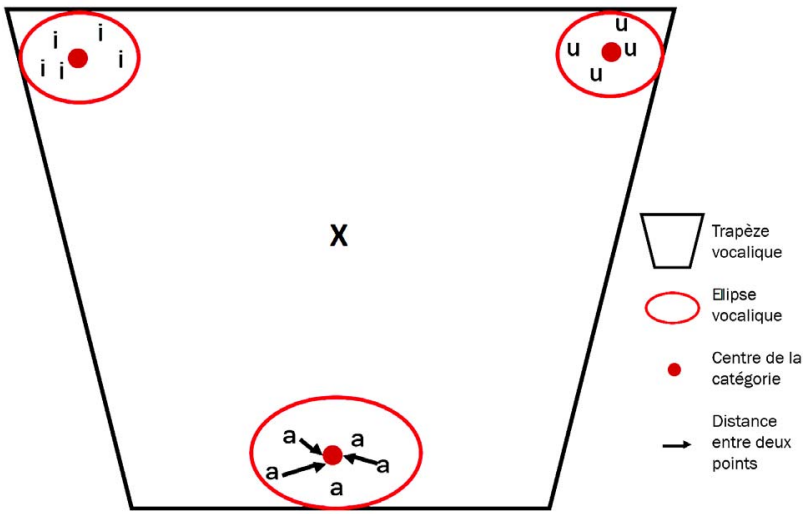

Fig. 4. Schématisation simplifiée de la méthode de calcul de la distance d'un exemplaire par rapport au centre de la catégorie vocalique à laquelle il appartient phonologiquement.

\subsection{Modèles statistiques}

Pour l'analyse quantitative des données nous avons fait le choix d'un modèle linéaire mixte, principalement parce que les contextes consonantiques n'étaient pas assez contrôlés pour être considérés comme des effets fixes d'une ANOVA (comme c'était le cas dans l'étude de Gahl [2015]). Nous avons donc utilisé la fonction lmer du package R lme4. La catégorie vocalique, le type de relation et type de tâche ont été intégrés au modèle en tant qu'effets fixes, tandis que le locuteur et le contexte consonantique ont été ajoutés comme effets aléatoires, la variable indépendante étant la métrique (avec un modèle par métrique). Des tests post-hoc (Tuckey HSD) ont été réalisés pour chaque métrique et chaque variable indépendante.

Comme conseillé par Nakagawa et Schielzeth [2013], nous avons calculé les valeurs $\mathrm{R}^{2}$ (marginales), représentant la variance expliquée, qui permet d'obtenir des valeurs absolues indiquant à quel point la variable indépendante testée influence la variable dépendante testée. Dans notre cas, cette valeur servira à nuancer le poids de certains effets sur la variation.

\section{Analyses et Résultats}

\subsection{La durée}

Les analyses montrent un effet global de la condition de production $(\mathrm{p}<0.001)$, de la locutrice $(\mathrm{p}<0.001)$, de la catégorie phonémique $(\mathrm{p}<0.001)$ et du contexte consonantique $(\mathrm{p}<0.001)$.

Les mesures $\mathrm{R}^{2}$ associées à nos modèles mixtes montrent qu'une proportion de $5.3 \%$ de la variation est liée à la condition de production $\left(\mathrm{R}^{2}=0.053\right), 5.2 \%$ à la locutrice $\left(\mathrm{R}^{2}=0.052\right)$ 
et $4.9 \%$ au contexte phonétique de la voyelle $\left(\mathrm{R}^{2}=0.049\right) ; 1.9 \%$ de la variation est explicable par la catégorie phonémique de la voyelle $\left(\mathrm{R}^{2}=0.019\right)$.

Les tests post-hoc montrent une différence significative des durées des voyelles entre la condition de lecture et les deux conditions de parole en interaction $(\mathrm{p}<0.001)$, avec des durées plus longues pour la liste de mots (moy=0.118; $\sigma=0.045)$, ainsi qu'une différence entre la conversation guidée faite avec un enquêteur $(\operatorname{moy}=0.079 ; \sigma=0.040)$ et la libre faite entre deux membres de la même famille (moy $=0.077 ; \sigma=0.039$ ), la première montrant des durées plus longues que la seconde $(\mathrm{p}=0.03)$.

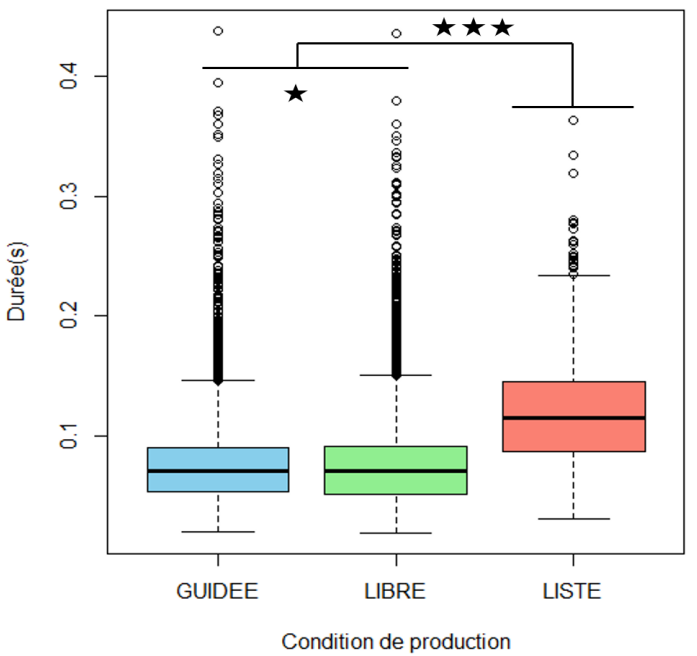

Fig. 5. Boites à moustache représentant la dispersion des valeurs de durée de chaque voyelle analysée en fonction de la tâche de production.

\subsection{La distance au centre du système}

Les analyses de distance au centre du système montrent un effet global de la condition de production $(\mathrm{p}<0.001)$, de la locutrice $(\mathrm{p}<0.001)$, de la catégorie phonémique $(\mathrm{p}<0.001)$ et du contexte consonantique $(\mathrm{p}<0.001)$.

Les mesures de taille d'effet associées à nos modèles mixtes montrent que $2.7 \%$ de la variation est liée à la condition de production $\left(\mathrm{R}^{2}=0.027\right), 3.1 \%$ à la locutrice $\left(\mathrm{R}^{2}=0.031\right)$ et $0.9 \%$ au contexte phonétique de la voyelle $\left(\mathrm{R}^{2}=0.009\right) ; 13 \%$ de la variation est explicable par la catégorie phonémique de la voyelle $\left(\mathrm{R}^{2}=0.132\right)$.

Les tests post-hoc montrent une différence significative entre la centralisation pour la liste de mot et celle pour les deux conditions de parole en interaction $(\mathrm{p}<0.001)$, avec un espace vocalique plus étendu pour la lecture de la liste de mots (moy=2.44; $\sigma=0.81$ ). La différence dans le degré de centralisation des condition «libre » $(\mathrm{moy}=1.992 ; \sigma=0.8)$ et « guidée » $(\operatorname{moy}=1.999 ; \sigma=0.78)$ n'était, elle, pas significative $(\mathrm{p}=0.533)$. 


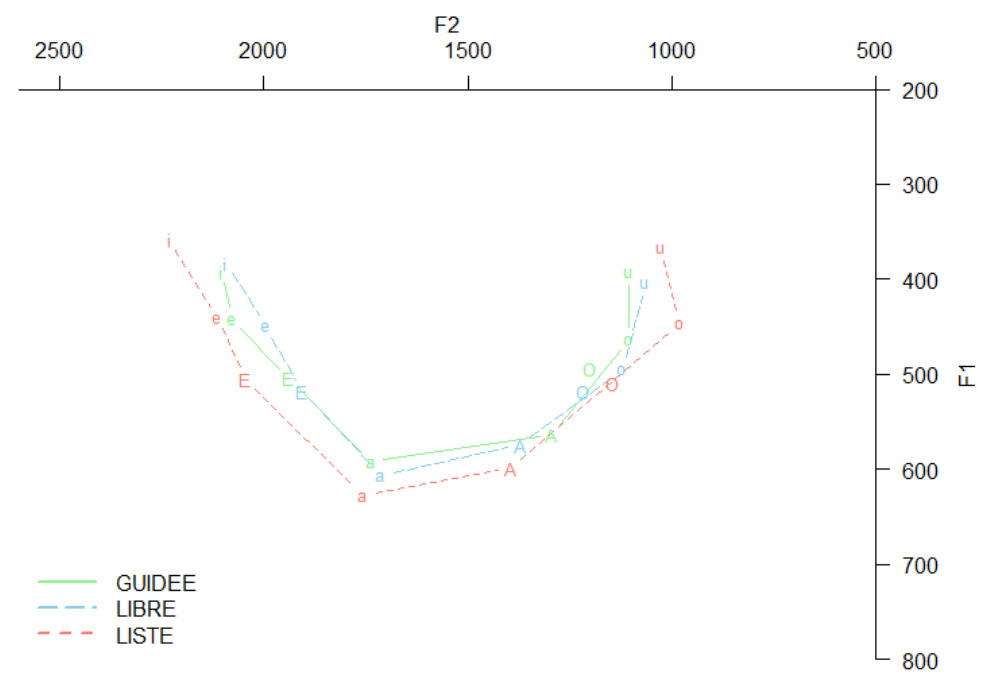

Fig. 6. Espace vocalique F1-F2 (en Hertz sur cette figure) pour chaque style de parole analysé.

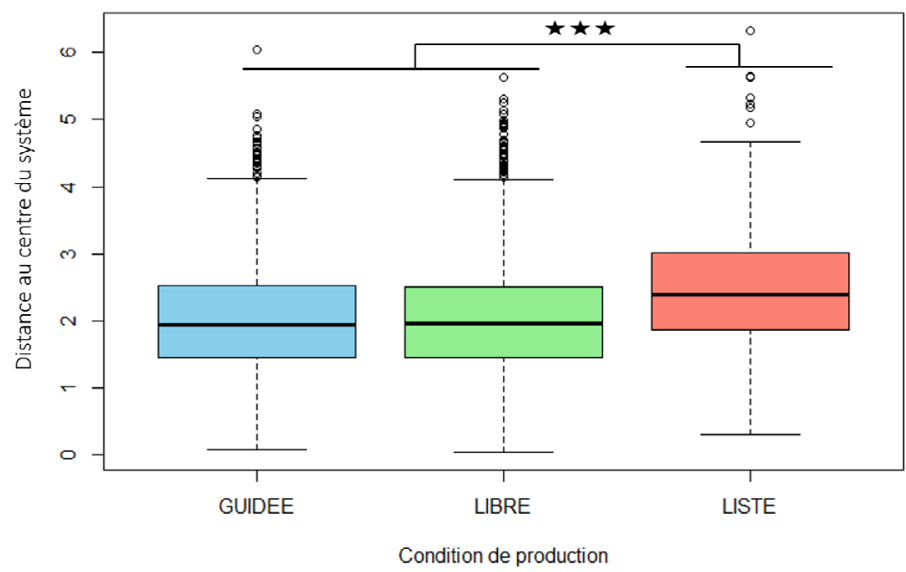

Fig. 7. Boites à moustache représentant la dispersion des valeurs de distance par rapport au centre du système vocalique pour chaque voyelle analysée en fonction de la tâche de production. L'axe des ordonnées donne un coefficient représentant l'éloignement des voyelles par rapport au centre du système vocalique.

\subsection{La dispersion intra-catégorie}

Les analyses par modèle mixte montrent un effet global de la condition de production $(\mathrm{p}<0.001)$, de la locutrice $(\mathrm{p}<0.001)$, de la catégorie phonémique $(\mathrm{p}<0.001)$ et du contexte consonantique $(\mathrm{p}<0.001)$.

Les mesures de taille d'effet associées à nos modèles mixtes montrent que $0.5 \%$ de la variation est liée à la condition de production $\left(\mathrm{R}^{2}=0.005\right), 0.6 \%$ à la locutrice $\left(\mathrm{R}^{2}=0.006\right)$, $0.5 \%$ au contexte phonétique de la voyelle $\left(\mathrm{R}^{2}=0.005\right) ; 1.4 \%$ de la variation est explicable par la catégorie phonémique de la voyelle $\left(\mathrm{R}^{2}=0.014\right)$.

Les tests post-hoc montrent une différence significative entre les dispersions intracatégories pour la conversation libre $(\operatorname{moy}=0.99 ; \sigma=0.61)$ et la conversation guidée $(\mathrm{p}<0.001)$ ainsi que pour la discussion libre et la lecture de la liste $(\mathrm{p}<0.001)$. La lecture de 
la liste de mot $(\operatorname{moy}=0.88 ; \sigma=0.56)$ et la discussion guidée (moy $=0.90 ; \sigma=0.54)$ montrent également des différences de dispersion $(\mathrm{p}<0.01)$.

Les figures 8 et 9 illustrent cette plus grande variation au sein des catégories dans le cas de la discussion avec un membre de la famille (libre) et une plus petite dispersion dans le cas de la lecture de la liste de mots (liste).

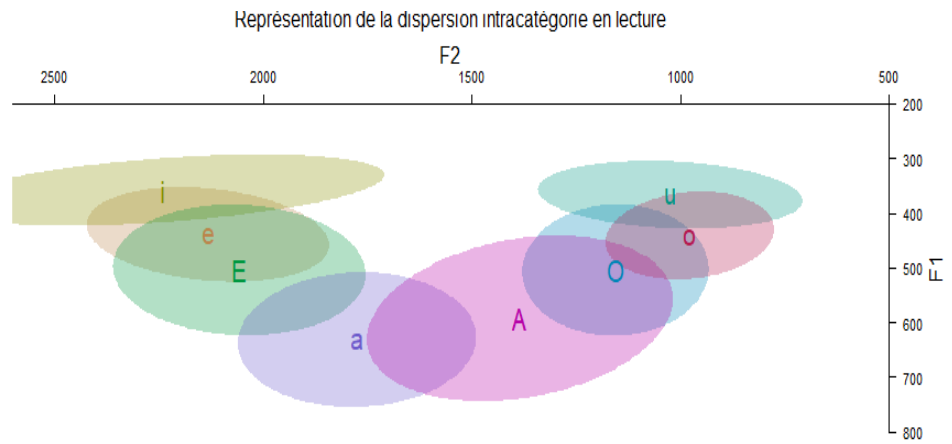

Représentation de la dispersion intracatégorie en conversation guidée
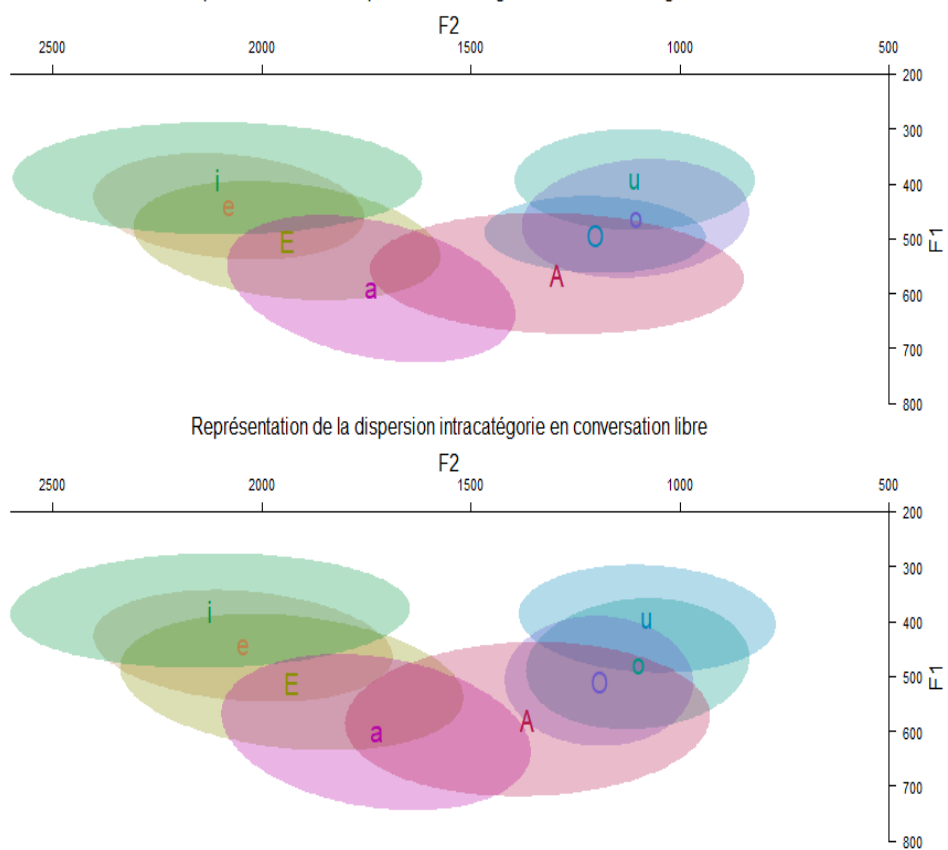

Fig. 8. Représentation de la dispersion intra catégorie dans l'espace F1-F2 (en Hertz) pour les trois styles de production. 


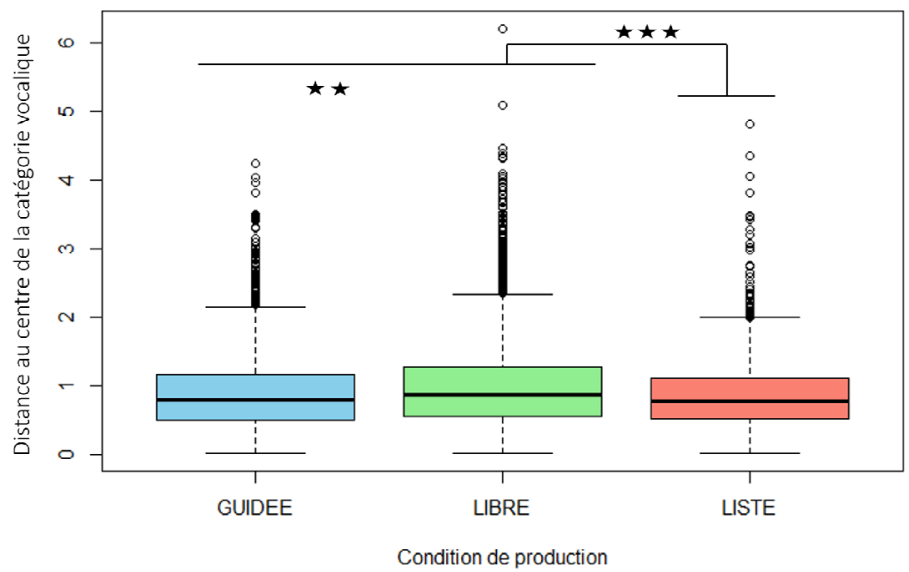

Fig. 9. Boites à moustache représentant la dispersion des valeurs de distance par rapport au centre des catégories phonémiques pour chaque voyelle analysée en fonction de la tâche de production. L'axe des ordonnées donne un coefficient représentant l'éloignement des voyelles par rapport au centre de leur catégorie vocalique.

\subsection{La diffusion}

Toutes catégories vocaliques mélangées, on constate un effet global de la condition de production $(\mathrm{p}<0.001)$, de la locutrice $(\mathrm{p}<0.001)$, de la catégorie phonémique $(\mathrm{p}<0.001)$ et du contexte consonantique $(\mathrm{p}<0.001)$.

Les mesures de taille d'effet associées aux modèles mixtes montrent que $0.2 \%$ de la variation dans l'écart entre les deux premiers formant (ici appelé « diffusion ») est liée à la condition de production $\left(\mathrm{R}^{2}=0.002\right), 0.3 \%$ à la locutrice $\left(\mathrm{R}^{2}=0.003\right)$ et $0.8 \%$ au contexte phonétique de la voyelle $\left(\mathrm{R}^{2}=0.008\right) ; 69 \%$ de la variation est explicable par la catégorie phonémique de la voyelle $\left(\mathrm{R}^{2}=0.693\right)$.

Les tests Post-hoc montrent une différence significative entre tous les styles $(p<0.001)$. La lecture de la liste de mots comporte les voyelles les plus diffuses, avec une distance entre le F1 et le F2 plus grande (moy=1317; $\sigma=566)$, suivie par la discussion avec l'enquêteur $(\mathrm{moy}=1276 ; \sigma=454)$; la discussion entre membres de la même famille comporte, elle, les voyelles les plus compactes (moy=1217; $\sigma=467)$.

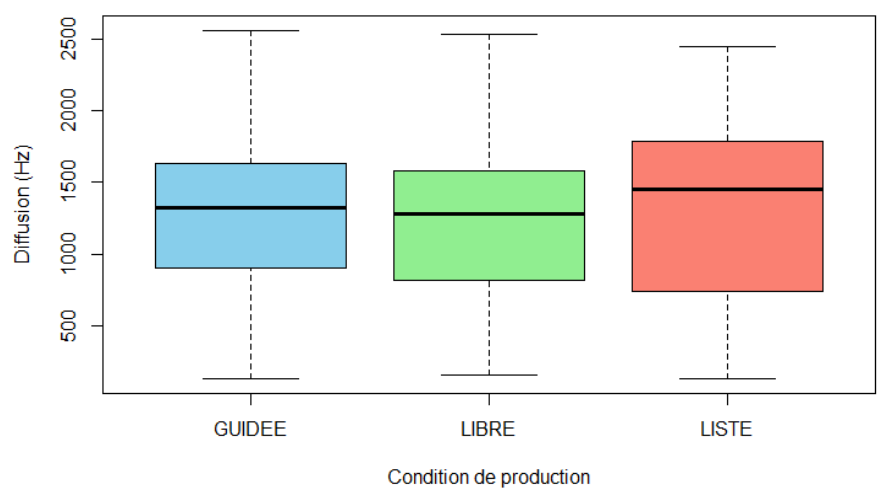

Fig. 10. Boites à moustache représentant la dispersion des valeurs de diffusion (écart entre F1 et F2, ici en Hertz) pour chaque voyelle analysée en fonction de la tâche de production. 
Pour éliminer le poids lié à la diffusion intrinsèque des voyelles, nous avons également analysé le comportement des voyelles intrinsèquement compactes $(/ \mathrm{a}, \mathrm{o}, \mathrm{J}, \mathrm{u} /)^{)}$et des voyelles intrinsèquement diffuses $(/ \mathrm{i}, \mathrm{e}, \varepsilon /)$ dans nos trois styles de parole.

Pour les compactes, nous observons une différence significative entre la guidée et la libre $(p<0.01)$, entre la guidée et la liste $(p<0.01)$ et entre la libre et la liste $(p<0.01)$. Pour les diffuses, on constate également cette différence significative entre les trois styles $(p<0.01)$. Les voyelles diffuses sont donc plus compactes et les voyelles compactes plus diffuses en parole spontanée qu'en lecture, et en discussion avec un inconnu plus qu'en discussion avec un membre de la famille.

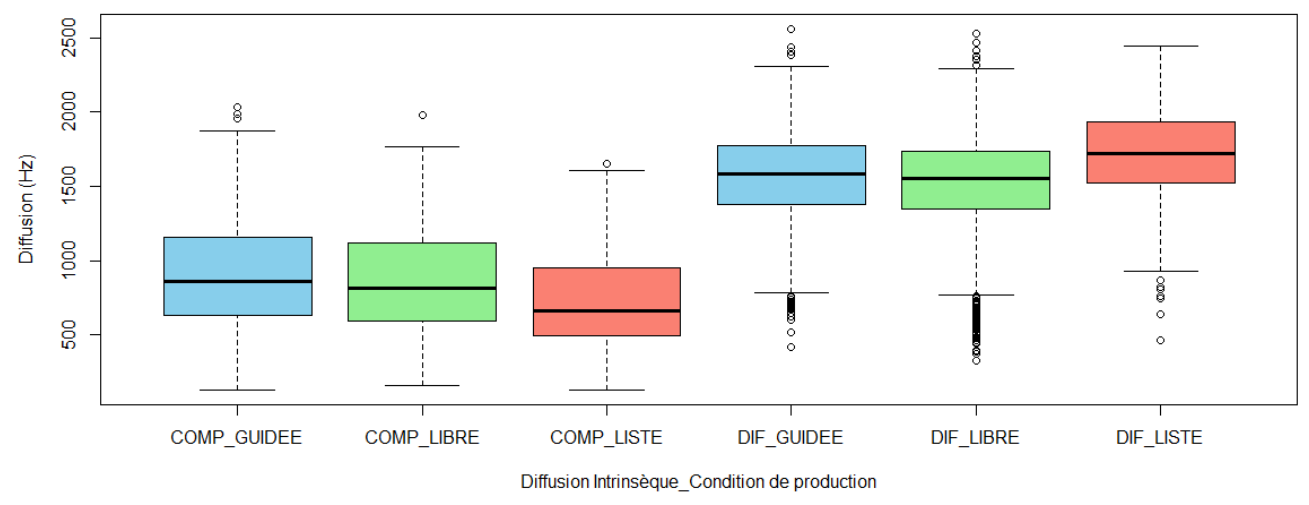

Fig. 11. Boites à moustache représentant la dispersion des valeurs de diffusion (écart entre F1 et F2) pour chaque voyelle analysée en fonction de la tâche de production et de sa diffusion intrinsèque.

\section{Conclusions et discussion}

L'analyse de notre sous-corpus tiré du projet PFC-Canada a permis de répliquer les résultats obtenus sur le français standard et de les pousser plus loin sur un plan sociophonétique. En plus de confirmer les différences de réduction spectrale entre la parole lue et la parole spontanée, nous avons mis en évidence l'importance de l'identité de l'interlocuteur et de la proximité relationnelle impliquée dans la discussion.

Nous avons relevé un schéma de réduction pour chacun des styles : la conversation dite libre (discussion avec une sœur ou une nièce) et la conversation dite guidée (discussion avec un enquêteur inconnu) montrent des voyelles plus courtes que la lecture, un système vocalique globalement plus centralisé, une dispersion intra-catégorie plus forte, des voyelles compactes plus diffuses et des voyelles diffuses plus compactes. Quant aux deux types de conversations, pour lesquels seul l'interlocuteur varie, nous observons une plus forte dispersion intra-catégorie pour la conversation avec un membre de la famille que pour la conversation avec un inconnu, et des voyelles compactes plus diffuses ainsi que des voyelles diffuses plus compactes.

Les mesures de taille d'effet montrent cependant que l'effet du style est souvent très léger par rapport à d'autres variables telles que la catégorie vocalique ou la locutrice (fig.12). Le corpus PFC-Canada étant particulièrement dense, nous prévoyons enrichir l'étude d'un nombre significatif de locuteurs, ce qui permettra d'avoir une meilleure idée de la taille de l'effet du locuteur et de le comparer à celui du style.

Nos résultats sont globalement congruents avec ceux des études de Harmegnies et Poch-Olivé [1992 ; 1994] pour la comparaison entre lecture et parole spontanée, ainsi qu'avec ceux de Scarborough et Zellou [2013a], qui avaient initié une comparaison en fonction de l'interlocuteur (ami/malentendant/absent/présent). Nous notons cependant que 
dans les résultats de Scarborough [2013], le degré d'hypoarticulation de la parole dirigée vers un ami n'est pas significativement différent de celui de la forme de citation des mots. Nous suggérons que cette différence entre nos résultats et ceux de Scarborough et Zellou [2013] est en partie liée à l'écologie de la situation de parole. L'étude citée élicitait la parole dirigée vers un ami par une consigne « prononcez ces phrases comme vous le feriez avec un ami » et le matériel prononcé était hautement contrôlé sur le plan phonétique, diminuant ainsi la validité écologique des données. Une autre hypothèse serait la différence de taille du corpus. En effet, nous avons analysé plus de 9000 segments, contre 1266 pour Scarborough et Zellou [2013], ce qui a peut-être permis de mettre en évidence des différences plus fines. Une dernière hypothèse serait que cette différence est liée à la langue : l'anglais pourrait ne pas montrer de différence d'hypoarticulation liée au statut relationnel, contrairement au français (laurentien).

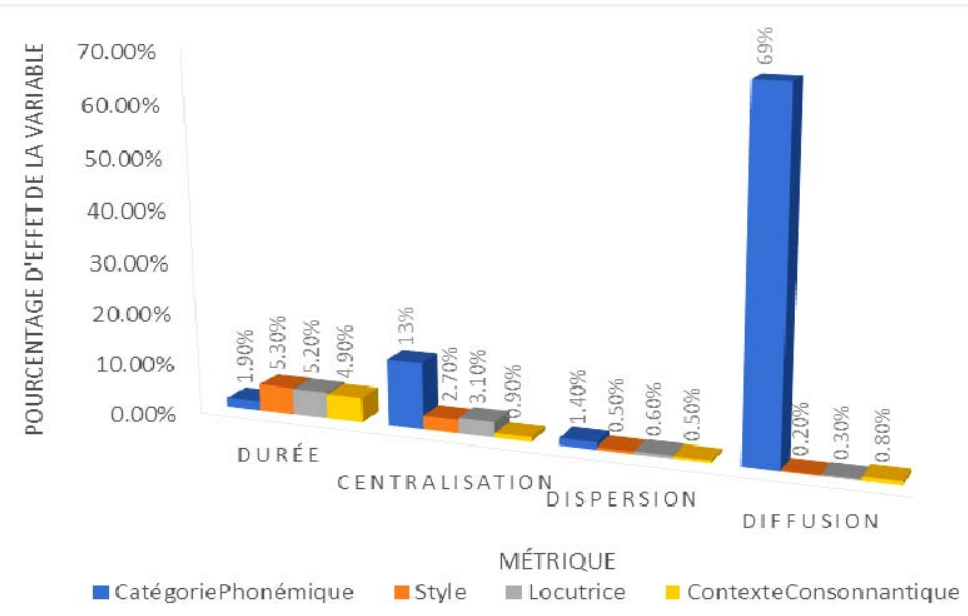

Fig. 12. Représentation en barres de la taille d'effet de chaque variable sur les métriques mesurées (valeurs $\mathrm{R}^{2}$ marginales en $\%$ ).

Il est nécessaire de poursuivre les analyses pour mieux comprendre les schémas de réduction de chaque style et l'interaction entre les métriques. Notamment le fait que la durée ne soit pas toujours corrélée au degré de centralisation, et la corrélation ou l'absence de corrélation entre les trois autres métriques.

Notre étude souffre également d'un manque de représentativité en ce qui concerne le nombre de catégorie vocaliques étudiées. L'ajout de données supplémentaires permettra de recueillir plus d'exemplaires de plus de catégories phonémiques, permettant une meilleure appréhension du comportement de chaque catégorie dans les différents styles abordés. Une autre étape de ce travail consisterait à prendre en compte la prosodie de façon plus satisfaisante, notamment en contrastant les positions syllabiques par rapport au mot (prosodique).

Enfin, un dernier problème surgit en français laurentien : la diphtongaison des /e/ et des /o/ en finale de mot. Nous avons qualitativement constaté ce phénomène dans les données du sous-corpus présenté ici, mais n'en avons pas tenu compte dans les analyses. Pour ces cas particuliers il serait intéressant d'adopter une méthodologie proche de celle de Van der Harst et collègues [2014] en prenant des mesures à cinq points différents pour chaque formant, ces cinq points permettant l'étude de la variation du formant au fil de la diphtongue. 
Nous remercions les participantes représentées dans cette étude et les enquêtrices et enquêteurs qui ont recueilli ces données (Karine Groulx, Élizabeth Morin-Lessard, Marie-Claude Séguin et, particulièrement, Hugo Saint-Amant Lamy), de même que les assistant·e·s qui ont contribué à la transcription des données.

\section{Références}

Audibert, N., Fougeron, C., Gendrot, C. \& Adda-Decker, M. (2015). Duration- vs. style-dependent vowel variation: a multiparametric investigation. In 18th International Congress of Phonetic Sciences, ICPhS'15, 5-10.

Bell, A. (1984). Language style as audience design. Language in Society, 13(2), 145-204.

Boersma, P. (2006). Praat : doing phonetics by computer. http://www. praat. org/

Bradlow, A. R., Kraus, N. \& Hayes, E. (2003). Speaking clearly for children with learning disabilities : sentence perception in noise. Journal of Speech, Language, and Hearing Research, 46(1), 80-97.

Côté, M-H. (2012). Laurentian French (Quebec) : extra vowels, missing schwas and surprising liaison consonants. In R. Gess, C. Lyche \& T. Meisenburg (éds), Phonological variation in French: Illustrations from three continents. Amsterdam : John Benjamins, 235-274.

Côté, M-H. (2014). Le projet PFC et la géophonologie du français laurentien. In J. Durand, G. Kristoffersen \& B. Laks, avec la collaboration de J. Peuvergne (éds), La phonologie du français : normes, périphéries, modélisation. Nanterre : Presses Universitaires de Paris Ouest, 175-198.

Durand, J., Laks, B. \& Lyche, C. (2002). La phonologie du français contemporain : usages, variétés et structure. In C. Pusch \& W. Raible (éds), Romanistische Korpuslinguistik - Korpora und gesprochene Sprache / Romance corpus linguistics - Corpora and spoken language. Tübingen : Gunter Narr, 93-106.

Erickson, D. (2002). Articulation of extreme formant patterns for emphasized vowels. Phonetica, 59(2-3), 134-149.

Eskenazi, M. (1993). Trends in speaking styles research. In Proceedings of Eurospeech-93, 501-509.

Ferguson, S. H. \& Kewley-Port, D. (2007). Talker differences in clear and conversational speech : acoustic characteristics of vowels. Journal of Speech, Language, and Hearing Research, 50(5), 1241-1255.

Flikeid, K. \& Cichocki, W. (1987). Application of dialectometry to Nova Scotia Acadian French dialects: phonological distance. In Papers from the 11th Annual Meeting of the Atlantic Provinces Linguistic Association. Université de Moncton, 59-74.

Fougeron, C., \& Audibert, N. (2011). Testing various metrics for the description of vowel distortion in dysarthria. In Actes de ICPhS 2011, 687-690.

Gahl, S. (2015). Lexical competition in vowel articulation revisited: vowel dispersion in the Easy/Hard database. Journal of Phonetics, 49, 96-116.

Harmegnies, B. \& Poch-Olivé, D. (1992). A study of style-induced vowel variability : laboratory versus spontaneous speech in Spanish. Speech Communication, 11(4-5), 429-437.

Harmegnies, B. \& Poch-Olivé, D. (1994). Formants frequencies variability in French vowels under the effect of various speaking styles. Journal de Physique IV 4.C5 (1994), C5-509-512.

Johnson, K. (2004). Massive reduction in conversational American English. In K. Yoneyama \& K. Maekawa (éds), Spontaneous speech : data and analysis. Proceedings of the 1st Session of the 10th International Symposium. Tokyo: The National International Institute for Japanese Language.

Labov, W. (1972). Sociolinguistic patterns. Philadelphie : University of Pennsylvania Press. 
Labov, W. (2002). Driving forces in linguistic change. Présenté à la International Conference on Korean Linguistics. Seoul National University, Séoul, Corée du Sud.

Léon, P (1993). Précis de phonostylistique. Paris : Armand Colin.

Lindblom, B. (1963). Spectrographic study of vowel reduction. Journal of the Acoustical society of America, 35(11), 1773-1781.

Lindblom, B. (1990). Explaining phonetic variation : a sketch of the H\&H theory. In W. J. Hardcastle \& A. Marchal (éds), Speech production and speech modelling. Dordrecht : Springer, 403-439.

Milne, P. (2014). The variable pronunciations of word-final consonant clusters in a force aligned corpus of spoken French. Thèse de doctorat, Université d'Ottawa.

Nakagawa, S. \& Schielzeth, H. (2013). A general and simple method for obtaining R2 from generalized linear mixed-effects models. Methods in Ecology and Evolution, 4(2), 133-142.

Nakamura, M., Koji, I. \& Sadaoki. F. (2008). Differences between acoustic characteristics of spontaneous and read speech and their effects on speech recognition performance. Computer Speech \& Language 22.2 (2008), 171-184.

Nguyen, N. \& Adda-Decker, M. (2013). Méthodes et outils pour l'analyse phonétique des grands corpus oraux. Paris : Hermes Science Publications.

Passy, P. (1917). Les sons du français. Paris : Firmin Didot.

Rouas, J. L. , Beppu, M. \& Adda-Decker, M. (2010). Comparaison des propriétés acoustiques de la parole lue, préparée et conversationnelle en français. In Actes des Journées d'Étude sur la Parole 2010, JEP2010, 245-250.

Rousselot, P. J. (1897-1901). Principes de phonétique expérimentale. Paris : H. Welter.

Scarborough, R., Dmitrieva, O., Hall-Lew, L., Zhao, Y., \& Brenier, J. (2007). An acoustic study of real and imagined foreigner-directed speech. Journal of the Acoustical Society of America, 121(5), 3044.

Scarborough, R. \& Zellou, G. (2013a). Clarity in communication : "clear" speech authenticity and lexical neighborhood density effects in speech production and perception. Journal of the Acoustical Society of America, 134(5), 3793-3807.

Scarborough, R. (2013b). Neighborhood-conditioned patterns in phonetic detail: relating coarticulation and hyperarticulation. Journal of Phonetics, 41(6), 491-508.

Tiffany, W. R. (1959). Nonrandom sources of variation in vowel quality. Journal of Speech and Hearing Research, 2, 305-317.

Van Bergem, D. R. (1993). Acoustic vowel reduction as a function of sentence accent, word stress, and word class. Speech Communication, 12(1), 1-23.

Van der Harst, S., Van de Velde, H. \& Van Hout, R. (2014). Variation in Standard Dutch vowels : the impact of formant measurement methods on identifying the speaker's regional origin. Language Variation and Change, 26(2), 247-272.

Walker, D. C. (1984). The pronunciation of Canadian French. Ottawa : University of Ottawa Press.

i Bien que la formule mesure l'éloignement d'un exemplaire à sa catégorie et non sa proximité, comme le laisse entendre le terme «centralisation ». 